LAWRENCE LIVERMORE N A T IO N A L LABORATORY

Integrated Chemical Systems: the Simultaneous Formation of Hybrid Nanocomposites of Iron Oxide and Organo Silsesquioxanes

L. Zhao, B.J. Clapsaddle, J.H. Satcher Jr., D.W. Schaefer, K.J. Shea

October 20, 2004

Chemistry of Materials 
This document was prepared as an account of work sponsored by an agency of the United States Government. Neither the United States Government nor the University of California nor any of their employees, makes any warranty, express or implied, or assumes any legal liability or responsibility for the accuracy, completeness, or usefulness of any information, apparatus, product, or process disclosed, or represents that its use would not infringe privately owned rights. Reference herein to any specific commercial product, process, or service by trade name, trademark, manufacturer, or otherwise, does not necessarily constitute or imply its endorsement, recommendation, or favoring by the United States Government or the University of California. The views and opinions of authors expressed herein do not necessarily state or reflect those of the United States Government or the University of California, and shall not be used for advertising or product endorsement purposes. 
Lihua Zhao , Brady J. Clapsaddle, Joe H. Satcher Jr.,

Dale W. Schaefer, Kenneth J. Shea

Chem Mater. 2004, 16, xxxx

Integrated Chemical Systems: the

Simultaneous Formation of Hybrid

Nanocomposites of Iron Oxide and Organo Silsesquioxanes
A sol-gel approach for the synthesis of hybrid nanocomposites of iron oxide and bridged polysilsesquioxanes has been developed. The hybrid nanocomposites exhibit a uniform dispersion of both components at length scales $>5 \mathrm{~nm}$.

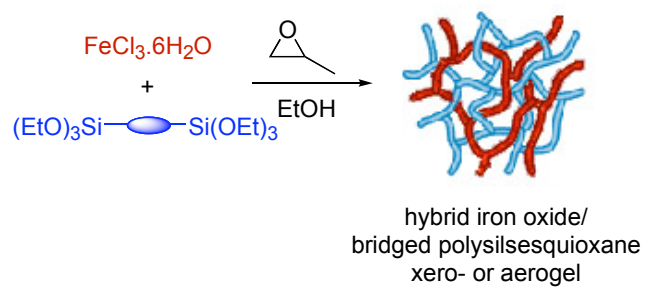




\title{
Integrated Chemical Systems: the Simultaneous Formation of Hybrid Nanocomposites of Iron Oxide and Organo Silsesquioxanes
}

\author{
Lihua Zhao $^{\dagger}$, Brady J. Clapsaddle ${ }^{\ddagger}$, Joe H. Satcher Jr. ${ }^{\ddagger}$, Dale W. Schaefer ${ }^{\S}$, Kenneth J. She ${ }^{\dagger *}$ \\ Department of Chemistry, University of California, Irvine, California 92697, Lawrence \\ Livermore National Laboratory, Livermore, California 94551, and Department of Materials \\ Science and Engineering, University of Cincinnati, Cincinnati, OH 45221-0012
}

\begin{abstract}
A sol-gel approach for the synthesis of hybrid nanocomposites of iron oxide and bridged polysilsesquioxanes has been established. The procedures allow for the simultaneous formation of iron oxide and polysilsesquioxane networks in monolithic xerogels and aerogels. These hybrid nanocomposites are synthesized from $\mathrm{FeCl}_{3} \cdot 6 \mathrm{H}_{2} \mathrm{O}$ and functionalized silsesquioxane monomers in a one-pot reaction using epoxides as a gelation agent. The porosity and microstructure of the materials has been determined by nitrogen porosimetry, electron microscopy and ultra small angle X-ray scattering (USAXS). The hybrid nanocomposites exhibit a uniform dispersion of both components with no evidence for phase separation at length scales $>5 \mathrm{~nm}$. At this limit of resolution it is not possible to distinguish between two independent interpenetrating networks integrated at molecular length scales or a random copolymer or mixtures of both.
\end{abstract}

\footnotetext{
*To whom correspondence should be addressed. Phone: 949-824-5844. Email: kjshea@uci.edu. $\dagger$ University of California, Irvine. \$Lawrence Livermore National Laboratory. §University of Cincinnati.
} 


\section{Introduction}

The integration of inorganic oxides with organics at nanometer length scales can extend the range of physical, mechanical, and chemical properties that can be obtained with simple mixtures of pure phases. ${ }^{1}$ To achieve mixing at these length scales, several strategies may be employed. One method involves the formation of interpenetrating networks. ${ }^{2}$ Interpenetrating networks (IPNs) may be assembled either sequentially or simultaneously, as depicted in Figure 1. The simultaneous formation of two or more interpenetrating networks is the most efficient. ${ }^{3}$ However, the challenge is to develop conditions where the rates of formation of two networks are approximately equal and the reaction conditions are compatible. There are several examples where this has been achieved. ${ }^{4}$

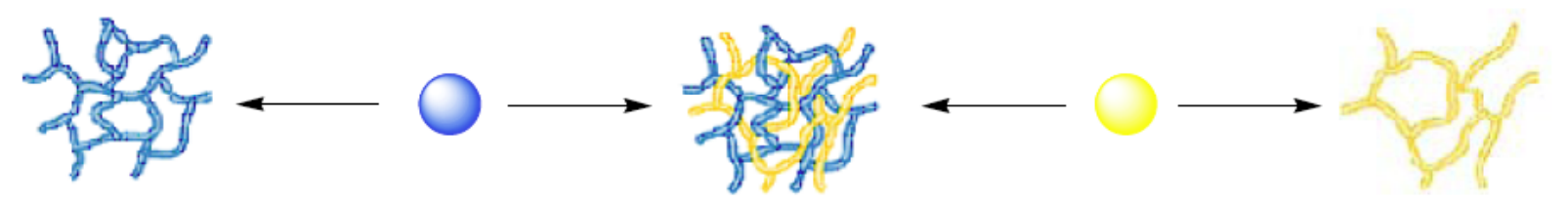

Figure 1. Illustration of independent and simultaneous network formation of two monomers.

The objective of this research is to develop methods for preparing monoliths of iron oxide and silsesquioxane networks that incorporate hydrocarbon or fluorocarbon groups as an integral part of the matrix. The organic component can be used to induce organization and/or long-range order, to modulate the mechanical properties, or enhance more specialized applications, such as their use as energetic materials. For these as well as other applications, it is desirable that both the inorganic and organic components be "mixed" at nanometer length scales.

Toward this end, we report the synthesis and characterization of hybrid monoliths comprised of iron oxide and polysilsesquioxanes. Two types of silsesquioxane precursors are included in this study, simple organo-functionalized triethoxysilanes and organo-bridged bistriethoxysilanes. These silsesquioxane precursors have different network forming efficiencies, so their relative ability to generate hybrid nanocomposites with iron oxides was also of interest. 
As such, this study establishes the compositional boundaries of the iron oxide and polysilsesquioxane components. Finally, several applications of these materials require the integration of perfluoroalkyl groups into the final hybrid material. Perfluoroalkyl group incorporation and dispersal in the matrix has also been accomplished.

Gash and co-workers have developed sol-gel procedures for preparing iron oxide xerogel and aerogel monoliths by the addition of epoxides to ethanol solutions of iron salts $\left(\mathrm{FeCl}_{3} \cdot 6 \mathrm{H}_{2} \mathrm{O}\right.$ or $\left.\mathrm{Fe}\left(\mathrm{NO}_{3}\right)_{3} \cdot 9 \mathrm{H}_{2} \mathrm{O}\right) .{ }^{5-6}$ In this system, the use of an epoxide as a proton scavenger was critical for controlling $\mathrm{pH}$ and preventing precipitation. The $\mathrm{pH}$ modulation afforded by the epoxide induces the Fe (III) species to undergo hydrolysis and slow, uniform condensation, as shown in Scheme 1.

Scheme 1

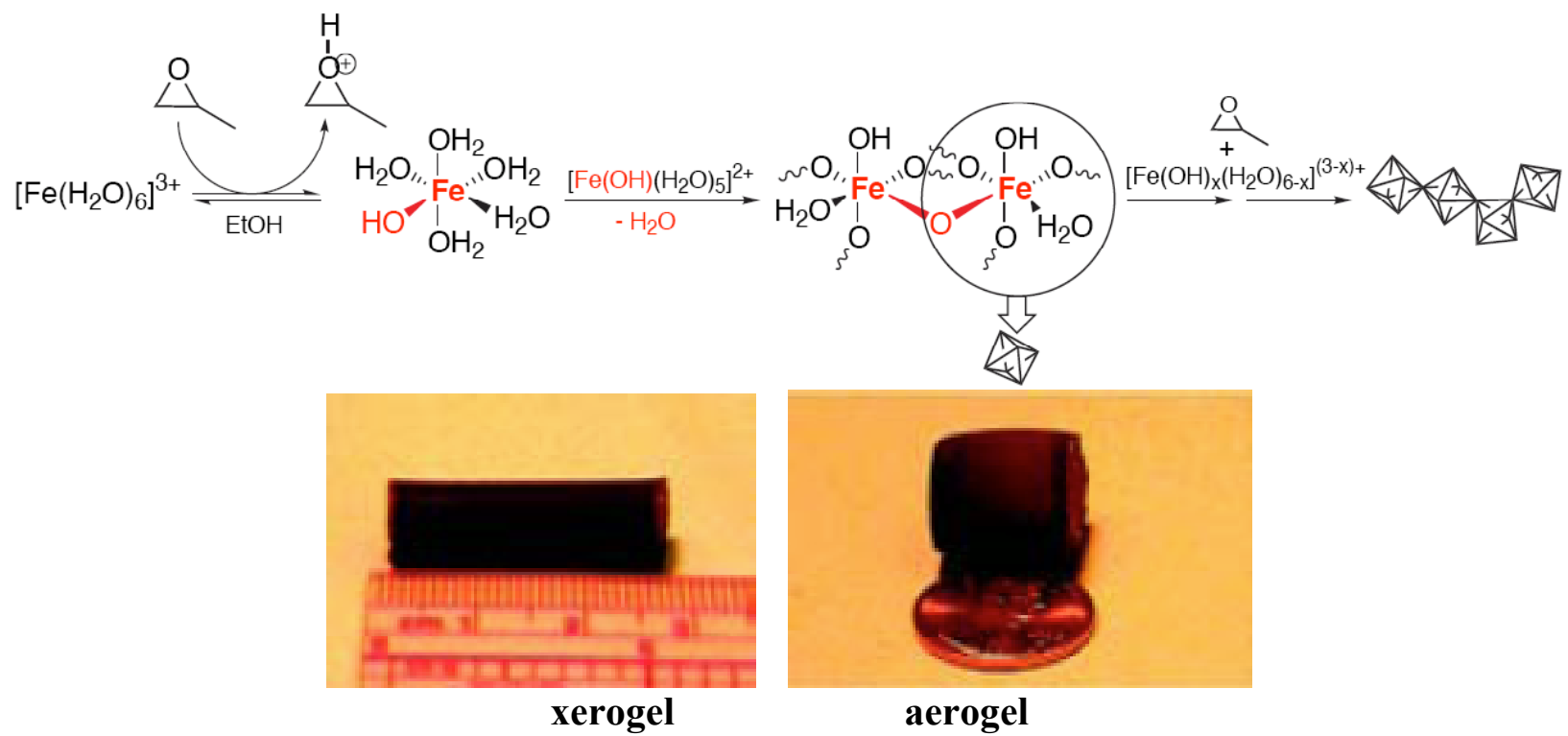

Their work was extended to the sol-gel synthesis of mixed Fe-Si oxide nanocomposite materials in which the iron oxide was the major component. ${ }^{7}$ These composite oxide materials were prepared in ethanol by inducing gelation of a mixture of $\mathrm{FeCl}_{3} \cdot 6 \mathrm{H}_{2} \mathrm{O}$ and either TEOS or TMOS through the addition of an epoxide. The composition of the resulting materials was varied from $\mathrm{Fe} / \mathrm{Si}(\mathrm{mole} / \mathrm{mole})=1-5$. 
Here we expand this technique to form hybrid iron oxide-polysilsesquioxane nanocomposites. These procedures allow the introduction of organics into the iron-silicon oxide matrix. A variety of organic functionalized silsesquioxane monomers, shown in Figure 2 (M1 M5), and $\mathrm{FeCl}_{3} \cdot 6 \mathrm{H}_{2} \mathrm{O}$ were gelled simultaneously in ethanol using the epoxide method just described. As with all complex multicomponent materials, control of domain size is important for tailoring and optimizing properties. The degree of integration of these two components was established by electron microscopy and ultra small angle x-ray scattering (USAXS).

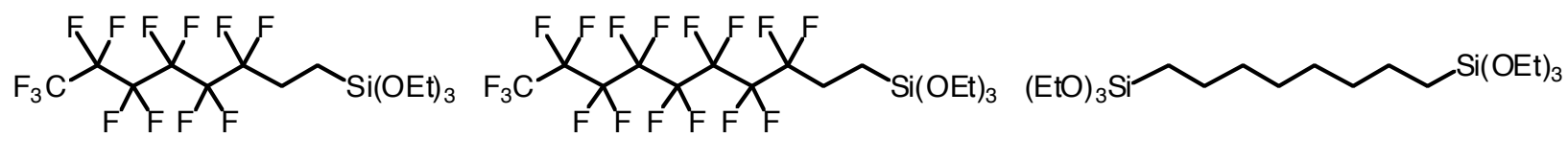

(M1)

(M2)

(M3)

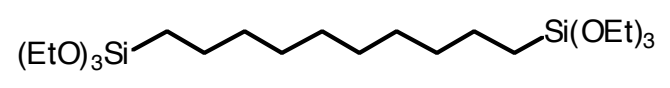

(M4)

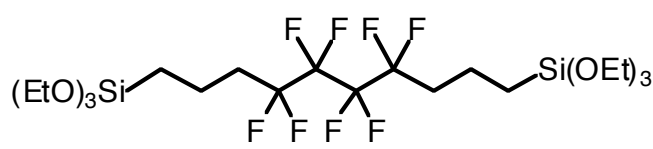

(M5)

Figure 2. The structure of organic functionalized silsesquioxane monomers used in this study.

\section{Experimental Section}

Materials. 1,4-Diiodo-perfluorobutane, allyl acetate, dibenzoyl peroxide (BPO), 1,10decadiene, triethoxysilane, $\mathrm{H}_{2} \mathrm{PtCl}_{6}$, propylene oxide (PO), trimethylene oxide (TMO) were purchased from Aldrich and used without further purification. (1-Triethoxysilyl-1,1,2,2tetrahydro)tridecafluorooctane (M1), (1-triethoxysilyl-1,1,2,2-tetrahydro)heptadecafluorodecane (M2) and bis(1,8-triethoxysily)octane (M3) were purchased from Gelest Inc. and used without further purification. Potassium bromide was purchased from VWR Scientific. Zinc powder was activated from commercially available zinc dust prior to use. ${ }^{8}$ Ethanol was distilled from a refluxed solution of magnesium turnings/magnesium ethoxide. Ferric chloride (lump, $\mathrm{FeCl}_{3}$. $6 \mathrm{H}_{2} \mathrm{O}$ ) was purchased from Fisher Scientific and used without further purification. Volatile solvents were removed under reduced pressure using a rotary evaporator, referred to as "removing solvent in vacuo". The abbreviations of chemical compounds and materials used in 
this paper are reported as follows: $\mathbf{M}=$ monomer, $\mathbf{X}=$ xerogel, $\mathbf{X F e}=$ iron(III) oxide xerogel, HG $=$ hybrid gel, $\mathbf{P O}=$ propylene oxide and TMO $=$ trimethylene oxide. All the numbers after the abbreviations correspond to the monomer number.

Synthesis of Silsesquioxane Monomers (M4 \& M5). 1,10-Bis(triethoxysilyl)decane (M4) was synthesized via hydrosilylation of 1,10-decadiene with triethoxysilane using $\mathrm{H}_{2} \mathrm{PtCl}_{6}$ as the catalyst, as described previously. ${ }^{9}$ Similarly, 1,10-bis(triethoxysilyl)-4,4,5,5,6,6,7,7octafluorodecane (M5) was synthesized via a three-step reaction using slightly modified literature procedures. ${ }^{10-13}$

Preparation of Polysilsesquioxane Xerogels (X4 \& X5) from M4 and M5. Synthesis of neat polysilsesquioxane xerogels has been previously reported. ${ }^{9,14}$ Briefly, decamethylenebridged polysilsesquioxane xerogels (X4) were synthesized from a solution of M4 (0.466 g, 1 $\mathrm{mmol})$ in THF $(0.95 \mathrm{~mL})$ to which was added $\mathrm{HCl}(0.11 \mathrm{~mL}, 1 \mathrm{~N})$ in THF $(0.95 \mathrm{~mL})$. After the vial was capped tightly, the resulting mixture was shaken for a few seconds, at which time the reaction became homogeneous. Gelation took place in approximately one minute. After aging for at least 2 days, the wet gel was soaked in water. The water was exchanged three times over a period of 3 days. The gel was then dried in the fume hood at room temperature for at least 15 days and $50{ }^{\circ} \mathrm{C}$ for 24 hours to afford the xerogel (X4). Octafluorodecamethlene-bridged polysilsesquioxane xerogels (X5) made with M5 $(0.305 \mathrm{~g}, 0.5 \mathrm{mmol})$ in THF $(0.50 \mathrm{~mL})$ and $\mathrm{HCl}$ $(54 \mu \mathrm{L}, 1 \mathrm{~N})$ in THF $(0.50 \mathrm{~mL})$ were synthesized and processed using the same procedures described for $\mathrm{X} 4$ xerogels.

Preparation of Iron(III) Oxide Xerogels (XFe) from $\mathrm{FeCl}_{3} \cdot \mathbf{6} \mathrm{H}_{2} \mathrm{O}$. Iron(III) oxide xerogels were prepared via a modified literature procedure. ${ }^{5-6}$ To a glass vial containing a fresh solution of $\mathrm{FeCl}_{3} \cdot 6 \mathrm{H}_{2} \mathrm{O}(0.840 \mathrm{mg}, 3 \mathrm{mmol})$ in absolute ethanol $(2.5 \mathrm{~mL})$ at $0{ }^{\circ} \mathrm{C}$, $\mathrm{PO}(1.45 \mathrm{~mL}$, $21 \mathrm{mmol}$ ) was gradually added in three aliquots over a period of $5 \mathrm{~min}$. (CAUTION: addition of $\mathrm{PO}$ to a $\mathrm{FeCl}_{3} \cdot 6 \mathrm{H}_{2} \mathrm{O}$ solution is accompanied by significant heat generation, which in some cases leads to flash boiling of the solution. The reaction should be carried out in a well-ventilated hood. The authors recommend the careful addition of $\mathrm{PO}$ to the $\mathrm{FeCl}_{3} \cdot 6 \mathrm{H}_{2} \mathrm{O}$ solution in two or 
three aliquots allowing for cooling time between additions.) After the complete addition of PO, the color of the solution changed from a clear yellow to an intense reddish-brown. The reaction mixture was briefly stirred and the stir bar was then removed. After the gel set in $\sim 5 \mathrm{~min}$, the glass vial was sealed with a cap and allowed to age for at least $24 \mathrm{~h}$.

Preparation of Hybrid Gels (HG1 \& HG2) from $\mathrm{FeCl}_{3} \cdot 6 \mathrm{H}_{2} \mathrm{O}$ and $\mathrm{M1}$ or M2. In a typical reaction, $\mathrm{FeCl}_{3} \cdot 6 \mathrm{H}_{2} \mathrm{O}(0.84 \mathrm{~g}, 3 \mathrm{mmol})$ was dissolved with stirring in absolute ethanol $(4.8 \mathrm{~mL})$ in a polyethylene vial to give a yellow-brown solution. Once the $\mathrm{FeCl}_{3} \cdot 6 \mathrm{H}_{2} \mathrm{O}$ was dissolved, the desired amount of neat $\mathbf{M} 1$ or $\mathbf{M} 2(0.6-0.1 \mathrm{mmol})$ was added to the $\mathrm{FeCl}_{3} \cdot 6 \mathrm{H}_{2} \mathrm{O}$ solution to achieve a Fe/Si molar ratio of 3-5. The resulting mixture was then allowed to stir for a minimum of 10 minutes prior to the addition of the epoxide gelation agent For gels made with M1, TMO was used as the gelation agent and was added in a single aliquot (1.6 $\mathrm{g}, 28 \mathrm{mmol})$. For composites made with M2, PO was the gelation agent and was added in three aliquots $(0.4 \mathrm{~g}$ (7 mmol)/aliquot, $21 \mathrm{mmol}$ total) approximately 15-20 minutes apart. After addition of each aliquot, the solution was briefly stirred to ensure thorough mixing. Following the addition of the final aliquot of epoxide, the stir bar was removed, the reaction vial covered, and the solution allowed to gel.

Preparation of Hybrid Gels (HG3) from $\mathbf{F e C l}_{3} \cdot \mathbf{6 H} \mathbf{H}_{\mathbf{2}} \mathrm{O}$ and $\mathrm{M3}$. $\mathrm{FeCl}_{3} \cdot 6 \mathrm{H}_{2} \mathrm{O}(0.840 \mathrm{~g}, 3$ mmol) was dissolved while stirring in absolute ethanol $(3.10 \mathrm{~mL})$ in a polyethylene vial. Simultaneously, the desired amount of M3 needed to achieve a Fe/Si atomic molar ratios of 0.5$5(0.6-6.0 \mathrm{mmol})$, was dissolved in absolute ethanol $(3.10 \mathrm{~mL})$ in a separate vial. Once the $\mathrm{FeCl}_{3} \cdot 6 \mathrm{H}_{2} \mathrm{O}$ was dissolved, the two solutions were combined and stirred for a minimum of 10 minutes. PO (1.60 g, $28 \mathrm{mmol})$ was then added to the mixture in two $0.8 \mathrm{~g}$ aliquots, allowing sufficient time for cooling between additions. Following the addition of the final aliquot of PO, the stir bar was removed, the reaction vial covered, and the solution allowed to gel.

Preparation of Hybrid Gels (HG4 \& HG5) from $\mathrm{FeCl}_{3} \cdot 6 \mathrm{H}_{2} \mathrm{O}$ and $\mathrm{M4}$ or M5. $\mathrm{FeCl}_{3} \cdot 6 \mathrm{H}_{2} \mathrm{O}(0.840 \mathrm{~g}, 3 \mathrm{mmol})$ was dissolved in absolute ethanol $(2.50 \mathrm{~mL})$ in a glass vial with a stir bar at $0{ }^{\circ} \mathrm{C}$. After simultaneous addition of neat M4 or M5 (Fe/Si atomic molar ratio of 3-6) 
and a portion of PO $(0.50 \mathrm{~mL})$, the reaction mixture was stirred, and the remaining PO was added in two aliquots $(0.50 \mathrm{~mL}$ and $0.45 \mathrm{~mL})$ over a period of $3 \mathrm{~min}$. The resulting mixture was briefly stirred for $1 \mathrm{~min}$, and the stir bar was then removed. The glass vial was sealed with a cap and the sol was allowed to gel.

Processing of Iron(III) Oxide and Hybrid Gels. All gels were covered and aged for at least $24 \mathrm{~h}$ at room temperature after the initial gelation. Each gel was then subjected to a porewashing/solvent exchange step in absolute ethanol for $3-5$ days. During this time, the wash solution was replaced with fresh ethanol at least three times. For aerogel preparation, the solventexchanged gels were processed in a Polaron ${ }^{\mathrm{TM}}$ supercritical point dryer. The ethanol in the wet gels was exchanged with $\mathrm{CO}_{2}(l)$ over $3-4$ days at $\sim 12{ }^{\circ} \mathrm{C}$, after which the temperature of the vessel was ramped to $\sim 45^{\circ} \mathrm{C}$ while maintaining a pressure of $\sim 100$ bar. The vessel was then depressurized at a rate of $\sim 7 \mathrm{bar} / \mathrm{h}$. For xerogel preparation, the wet gels were covered with perforated aluminum foil and allowed to dry in a fume hood for 15 - 30 days at room temperature. The resulting xerogel monoliths were then placed in vacuum (20-10 mtorr) for $24 \mathrm{~h}$ to complete the drying process.

Physical Characterization. Surface area and pore volume and size analyses were performed using an ASAP 2000 porosimeter (Micromeritics Instrument Crop.). Sample amounts of 180-280 mg were used for analysis. Prior to analysis, the glassy, brittle xerogels were coarsely ground and degassed under vacuum (below 5 mtorr). Surface area determination and pore size and volume analysis were performed by BET (Brunauer-Emmett-Teller) and BJH (BarrettJoyner-Halenda) methods, respectively. ${ }^{15,16}$

Scanning electron microscopy (SEM) was carried out using a Hitachi S-4500 cold field emission microscope. Typical accelerating voltages used for xerogel samples ranged from $1 \mathrm{Kv}$ to $10 \mathrm{Kv}$ depending on magnification. Coating of samples with a conductive layer Au was necessary in some cases.

For sample HG5, transmission electron microscopy (TEM) was performed on a Philips CM20 microscope with a liquid nitrogen holder. Powered samples were dispersed in acetone and 
drops of the resulting mixture were deposited on a plasma-etched amorphous carbon substrate supported copper grid using a pipette. TEM for samples of HG1, HG2 and HG3 was performed on a Philips CM300FEG microscope operating at $300 \mathrm{keV}$ using zero loss energy filtering with a Gatan energy imaging filter (GIF) to remove inelastic scattering. The images were taken under bright field conditions and slightly defocused to increase contrast. The images were also recorded on a $2 \mathrm{~K} \times 2 \mathrm{~K} \mathrm{CCD}$ camera. Energy Filtered TEM (EFTEM) element maps were obtained by electron energy loss spectroscopy (EELS) in tandem with the Philips CM300FEG TEM microscope. All EELS measurements were made with a Gatan model 607-electron energyloss spectrometer attached to the microscope. Measurements were made at the Fe- $\mathrm{L}_{2,3}, \mathrm{O}-\mathrm{K}$, and $\mathrm{Si}-\mathrm{L}_{2,3}$ edges. Images were processed using Digital Micrograph ${ }^{\mathrm{TM}}$ 3.3.1 software from Gatan, Inc.

Fourrier transform infrared spectroscopy (FTIR) was performed on either a ThermoNicolet Nexus 4000 FTIR spectrometer or a Perkin \& Elmer FTIR System. Samples were prepared by grinding together $1 \mathrm{mg}$ of the organic/inorganic nanocomposite with $100 \mathrm{mg}$ of dry potassium bromide with a mortar and pestle. The mixture was pressed into a pellet using a standard bolt press prior to analysis. The spectra were collected in transmission mode at $5 \mathrm{~cm}^{-1}$ resolution for 32 scans.

Ultra small angle x-ray scattering (USAXS) was performed using the Bonse-Hart camera at the UNICAT beam line at the Advanced Photon Source, Argonne National Laboratory. Samples were prepared by placing $S \operatorname{cotch} \circledast$ magic tape over the bottle containing the sample (XFe, HG4, and HG5) and turning the bottle to allow the powder to stick to the tape. A barely visible layer of powder on the tape was then covered with a second piece of magic tape. Two pieces of tape with no sample were used as a background that is subtracted from all data reported here. The data were desmeared using routines provided by UNICAT. 


\section{Results and Discussion}

Monomer Synthesis. The synthesis of both M4 and M5 is illustrated in Scheme 2. The monomer 1,10-bis(triethoxysilyl)decane, M4, was synthesized from 1,10-decadiene and triethoxysilane via hydrosilylation using $\mathrm{H}_{2} \mathrm{PtCl}_{6}(0.03 \mathrm{~mol} \%)$ as catalyst. ${ }^{9}$ The fluorinated monomer 1,10-bis(triethoxysilyl)-4,4,5,5,6,6,7,7-octafluorodecane, M5, was synthesized using a $\mathrm{H}_{2} \mathrm{PtCl}_{6} \quad(0.3 \mathrm{~mol} \%)$ catalyzed hydrosilylation reaction between triethoxysilane and 4,4,5,5,6,6,7,7-octafluoro-1,10-decadiene, the latter being prepared via a two step reaction involving acetylation of 1,4-diiodoperfluorobutane followed by elimination of diacetate to form the fluorinated diene. ${ }^{10-13}$

\section{Scheme 2}

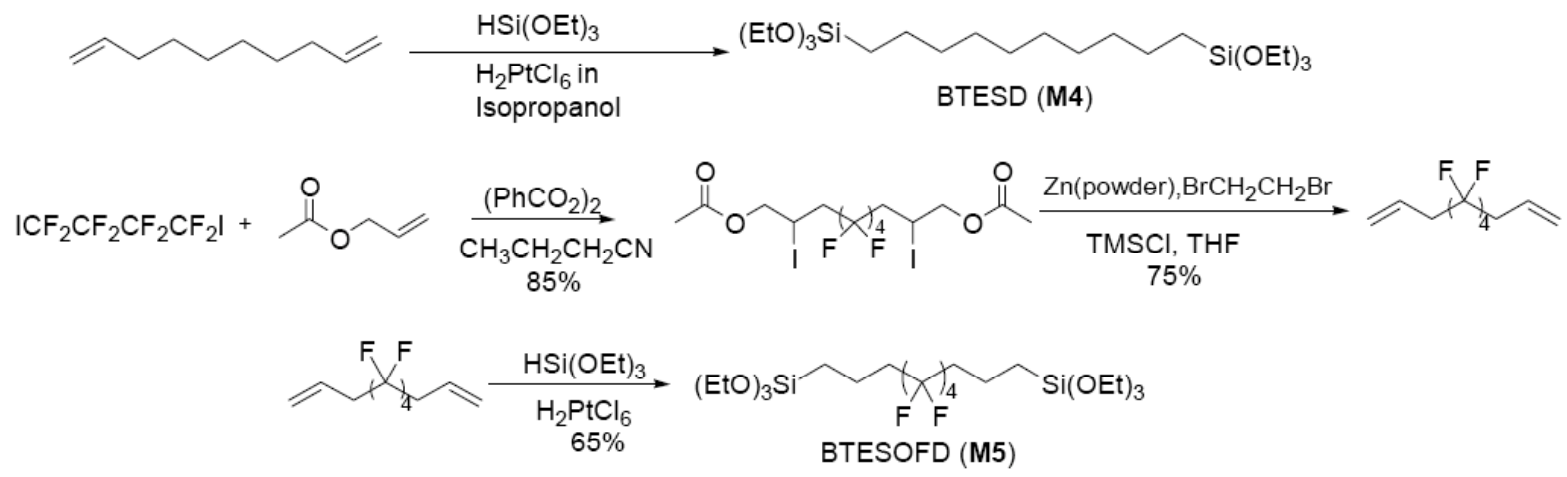

Material Synthesis. Pure iron oxide xerogels, XFe, were prepared from solutions of $\mathrm{FeCl}_{3} \cdot 6 \mathrm{H}_{2} \mathrm{O}$ in ethanol using $\mathrm{PO}$ as an acid scavenger following the method of Gash et. al as illustrated above in Scheme 1. ${ }^{5-6}$ The gel time $\left(t_{\text {gel }}\right)$ for XFe materials was approximately 5 minutes. Following gelation, the material was aged and then processed by washing in ethanol for 3 - 5 days followed by ambient drying. The two polysilsesquioxane xerogels, $\mathbf{X} \mathbf{4}$ and $\mathbf{X 5}$, were synthesized via acid catalyzed sol-gel polymerization from solutions of M4 or M5 in THF using $1 \mathrm{M} \mathrm{HCl}$ as the catalyst, as shown in Scheme 3. ${ }^{9,14}$ At a monomer concentration of $0.4 \mathrm{M}$ the $t_{\mathrm{gel}}$ for both materials was less than 10 minutes. After aging for $48 \mathrm{~h}$, the materials were processed by water washing followed by ambient drying to produce xerogels. Although monomers M1 and M2 readily undergo hydrolysis and condensation under the conditions employed here, they do 
not independently form gels. The three xerogels, XFe, X4, and X5, were used as reference materials for comparison to the organic/inorganic hybrid systems.

\section{Scheme 3}

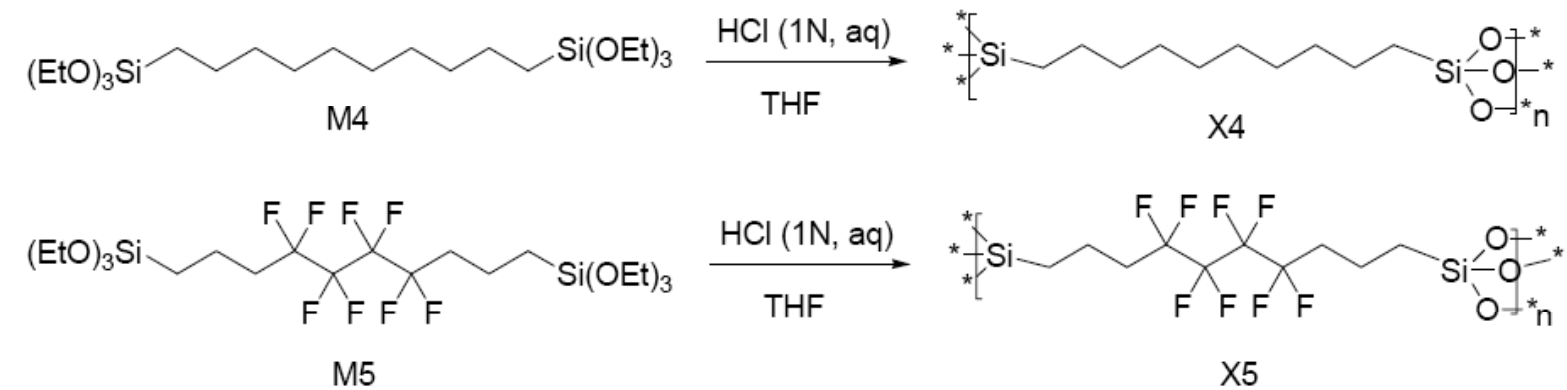

In order to establish optimal conditions for the simultaneous formation of the iron oxide and silsesquioxane networks in the synthesis of the hybrid materials, $t_{\text {gel }}$ for the individual networks under conditions of varying $\mathrm{pH}$ were noted. Although the $\mathrm{pH}$ of an ethanolic solution of $\mathrm{FeCl}_{3} \cdot 6 \mathrm{H}_{2} \mathrm{O}$ increases uniformly from $\sim 1.0$ to $\sim 5.2$ upon the addition of $\mathrm{PO}$, the strongly acidic solution during initial stages of the reaction is conducive to rapid silsesquioxane network formation. ${ }^{5,17,18}$ The $t_{\mathrm{gel}}$ of $\mathrm{FeCl}_{3} \cdot 6 \mathrm{H}_{2} \mathrm{O}$ solutions in ethanol with $\mathrm{PO}\left(\mathrm{k}_{1}\right)$ were comparable to the $t_{\text {gel }}$ of bridged monomers M4 and M5 in the concentration range of 0.1-0.4M under acidic conditions $\left(\mathrm{k}_{2}\right)$, suggesting that the simultaneous formation of the two networks could be achieved under these conditions (Scheme 4). Concentrations of the silsesquioxane monomers were chosen based on values that were anticipated for their incorporation into the iron (III) oxide matrix. The ratios and concentrations used corresponded to similar $t_{\text {gel }}$ for the independent systems. The hybrid materials were prepared from ethanol solutions of $\mathrm{FeCl}_{3} \cdot 6 \mathrm{H}_{2} \mathrm{O}$ and silsesquioxane monomers M1-M5 using epoxides as acid scavengers to establish pH gradients and induce gelation. This process is depicted in Scheme 5 along with an illustration of the resulting hybrid xerogel.

Hybrid gel materials, HG1 and HG2, prepared from monotriethoxy silsesquioxanes , were prepared from ethanol solutions of $\mathrm{FeCl}_{3} \cdot 6 \mathrm{H}_{2} \mathrm{O}$ and $\mathbf{M 1}$ or M2. Gelation of these materials 


\section{Scheme 4}

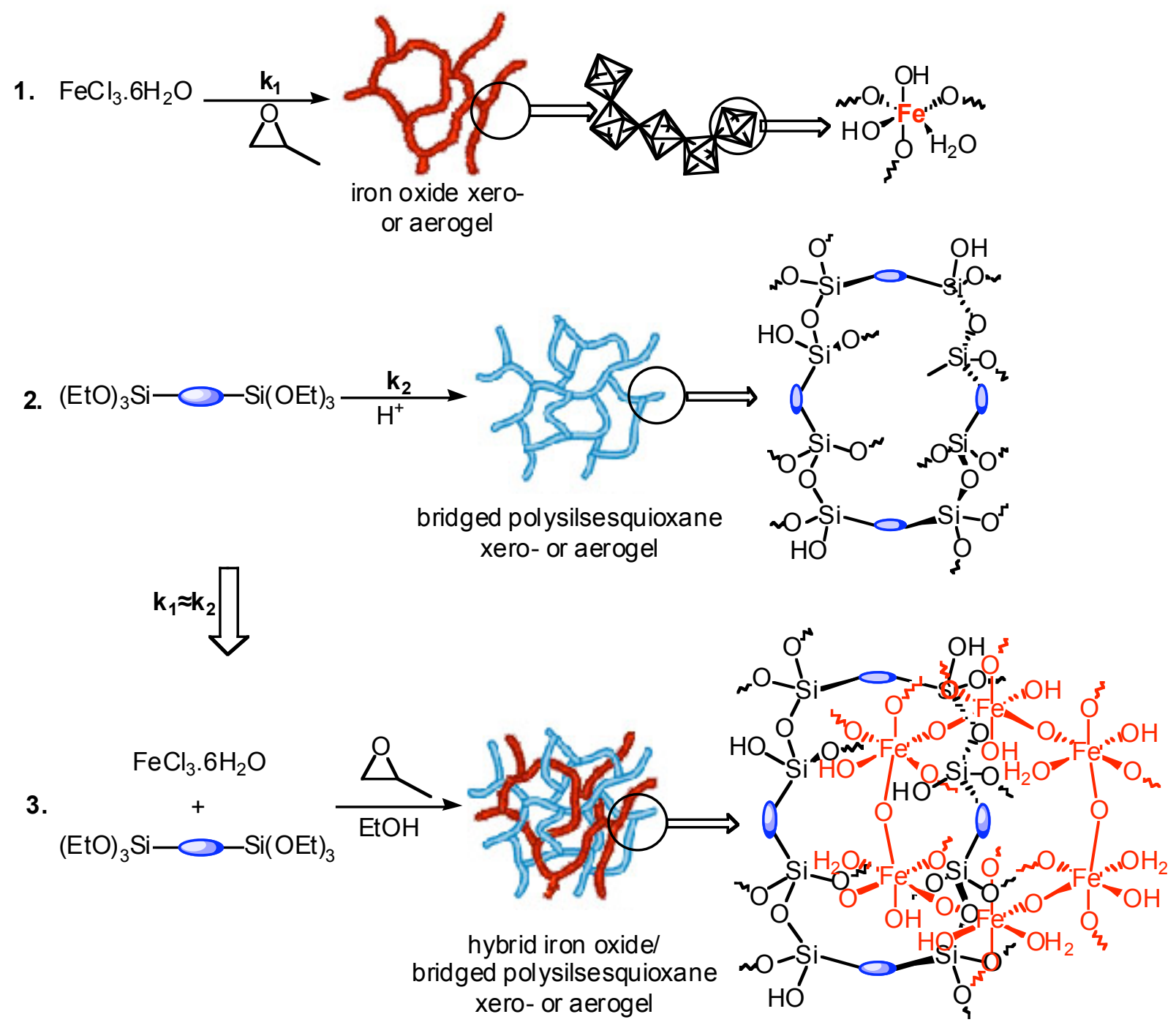

\section{Scheme 5}
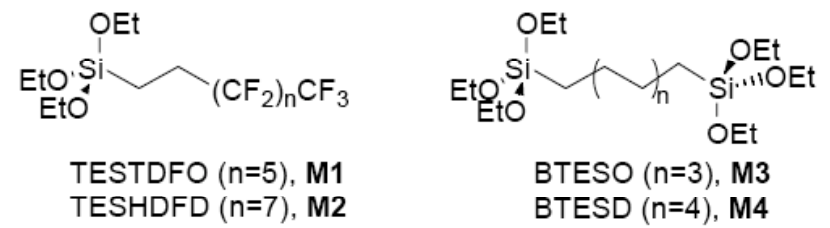

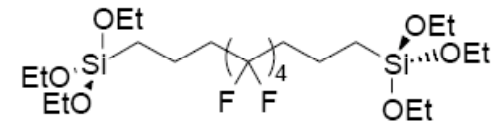

BTESOFD, M5

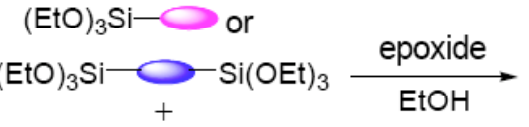

$$
\begin{aligned}
& \mathrm{FeCl}_{3} \cdot 6 \mathrm{H}_{2} \mathrm{O}
\end{aligned}
$$

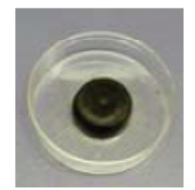


was achieved through the use of two different epoxides, PO or TMO (Table 1). The use of the different epoxides depended on the composition desired in the various HG1 or HG2 materials within the boundaries for maintaining a homogeneous solution up to gelation. Compositional studies with different $\mathrm{Fe} / \mathrm{Si}$ ratios revealed that $\mathrm{Fe} / \mathrm{Si}$ atomic molar ratios less than 5 resulted in formation of precipitates when M1 was the silsesquioxane and PO was the gelation agent. However, Fe/Si ratios could be lowered to 4 using TMO to prepare HG1. As can be seen in Table 1, the use of TMO as a gelation agent greatly increased the $t_{\text {gel }}$ for these materials due to the slower proton scavenging reaction of the four membered oxetane ring (TMO) versus the three membered epoxide ring (PO). ${ }^{5-7}$ The slower $t_{\text {gel }}$ allowed for a slight decrease in the Fe/Si ratio when using monomer M1. The HG2 materials could be made with both PO and TMO in ratios as low as $\mathrm{Fe} / \mathrm{Si}=3$. Efforts to synthesize hybrid gels with $\mathrm{Fe} / \mathrm{Si}<3$ using either $\mathbf{M 1}$ or M2 resulted in separation of the reaction solution into two distinct phases accompanied by formation of precipitates.

Table 1. Summary of preparation conditions for HG1 and HG2

\begin{tabular}{cccccc}
\hline material & monomer & calc. Fe/Si $(\mathrm{mol} / \mathrm{mol})$ & epoxide $^{\mathrm{a} .}$ & gel type & $t_{\text {gel }}$ \\
\hline HG1-1 & M1 & 4 & TMO & aero & $20 \mathrm{hr}$ \\
HG1-2 & M1 & 4 & TMO & xero & $20 \mathrm{hr}$ \\
HG2-2 & M2 & 5 & PO & aero & $6 \mathrm{~min}$ \\
HG2-2 & M2 & 5 & PO & xero & $6 \mathrm{~min}$. \\
HG2-3 & M2 & 3 & PO & aero & $18 \mathrm{~min}$. \\
\hline
\end{tabular}

a. For materials prepared with TMO, TMO/Fe ${ }^{3+}=9.3(\mathrm{~mol} / \mathrm{mol})$; for materials prepared with $\mathrm{PO}$, $\mathrm{PO} / \mathrm{Fe}^{3+}=7$.

Materials prepared from bistriethoxy silsesquioxanes, HG3, HG4, and HG5 were synthesized in a manner similar to that of HG1 and HG2. The conditions are summarized in Table 2. A neat silsesquioxane monomer and an aliquot of PO were simultaneously added to a 
solution of $\mathrm{FeCl}_{3} \cdot 6 \mathrm{H}_{2} \mathrm{O}(1.2 \mathrm{M})$ in ethanol at $0{ }^{\circ} \mathrm{C}$. For all gels, after the addition of the epoxide, the homogeneous solution changed color from red-orange to dark red-brown, and then turned into a monolithic red-brown gel within 10 minutes. Hybrids of HG3 were processed to both xerogels and aerogels and remained monolithic following processing. Compared to HG1 and

\begin{tabular}{|c|c|c|c|c|}
\hline material & monomer & calc. Fe/Si (mol/mol) & gel type & $t_{\mathrm{gel}}(\mathrm{min})$ \\
\hline XFe & None & --- & xero & 5 \\
\hline X4 & M4 & 0 & xero & $\sim 1$ \\
\hline $\mathbf{X 5}$ & M5 & 0 & xero & $<10$ \\
\hline HG3-1 & M3 & 2 & aero & 40 \\
\hline HG3-2 & M3 & 1 & aero & 75 \\
\hline HG3-3 & M3 & 0.5 & aero & 90 \\
\hline HG3-4 & M3 & 2.5 & xero & 40 \\
\hline HG3-5 & M3 & 1 & xero & 75 \\
\hline HG4-1 & M4 & 6 & xero & 5 \\
\hline HG4-2 & M4 & 3 & xero & 3 \\
\hline HG4-3 & M4 & 1 & xero & 1 \\
\hline HG4-4 ${ }^{\text {b. }}$ & M4 & 1 & xero & $>120$ \\
\hline HG5-1 & M5 & 6 & xero & 5 \\
\hline HG5-2 & M5 & 5 & xero & 7 \\
\hline HG5-3 & M5 & 3 & xero & 8 \\
\hline
\end{tabular}

a. All gels were prepared using PO as the epoxide. $\mathrm{PO} / \mathrm{Fe}^{3+}=7(\mathrm{~mol} / \mathrm{mol})$ for all materials accept

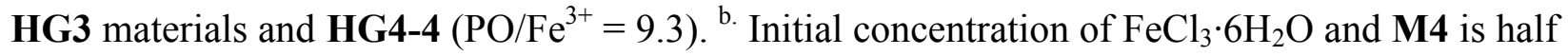
of that used for the other HG4 materials $\left(\left[\mathrm{FeCl}_{3} \cdot 6 \mathrm{H}_{2} \mathrm{O}\right] \approx 0.6 ;[\mathrm{M} 4] \approx 0.25\right)$. 
HG2, the synthesis of HG3, HG4, and HG5 materials could be achieved even when the Fe/Si ratios were as low as 0.5 . This could be due to the increased solubility of the bissilsesquioxanes compared to $\mathbf{M 1}$ and $\mathbf{M 2}$.

It is interesting to compare materials prepared from monomers M1 and M2 (HG1, HG2) with those from the difunctional monomers M3, M4, and M5 (HG3, HG4, and HG5). As can be seen in Tables 1 and 2, the organosilica content of the materials can be significantly increased when using the bissilsesquioxane starting materials. Phase separation and precipitation was not observed for the bissilsesquioxane systems for $\mathrm{Fe} / \mathrm{Si} \leq 3$, presumably due to the increased solubility of the bissilsesquioxanes in the ethanol synthesis solution. In fact, materials in which organosilica was the dominant phase $(\mathrm{Fe} / \mathrm{Si}<1)$ could be achieved when using the bissilsesquioxane starting materials. Hybrids prepared with bissilsesquioxane monomers can achieve higher loadings of organic. However, these hybrids will have a higher silicon content in the final material than those prepared with monosilsesquioxane precursors, a consequence that can have an effect on the properties of the final materials.

\section{Materials Characterization}

Nitrogen Adsorption/Desorption Analysis. The surface area, pore volume and average pore diameter were obtained by analysis of the nitrogen adsorption/desorption isotherms (Table 3). Comparison of the results for reference and IPN materials can be useful for evaluating the degree of interpenetration of the iron (III) oxide and polysilsesquioxane networks. Table 3 contains the surface area, pore volume, and average pore diameter for selected dried hybrid IPN materials. The hybrid materials are largely mesoporous with the surface areas ranging from 70-

$350 \mathrm{~m}^{2} / \mathrm{g}$. All xerogels have lower surface areas, pore volumes, and pore diameters than aerogels, as expected for the denser, glass-like structure of the ambiently dried xerogel materials. Analysis of these materials displayed the classic type IV isotherm, indicative of a mesporous material (pore diameters $2-50 \mathrm{~nm}$ ), and narrow pore size distributions. ${ }^{15,16}$ 
Table 3. Summary of surface area and porosities of hybrid gels

\begin{tabular}{cccccccc}
\hline materials & monomer & epoxide & $\begin{array}{c}\text { calc. } \\
\text { Fe/Si } \\
(\mathrm{mol} / \mathrm{mol})\end{array}$ & $\begin{array}{c}\text { gel } \\
\text { type }\end{array}$ & $\begin{array}{c}\text { surface } \\
\text { area }^{\mathrm{a}} \\
\left(\mathrm{m}^{2} / \mathrm{g}\right)\end{array}$ & $\begin{array}{c}\text { pore } \\
\text { volume }^{\mathrm{b}} \\
(\mathrm{mL} / \mathrm{g})\end{array}$ & $\begin{array}{c}\text { pore } \\
\text { diameter }^{\mathrm{c}} \\
(\mathrm{nm})\end{array}$ \\
\hline XFe & none & PO & N/A & xero & 378 & 0.27 & 26 \\
XFe-2 & none & PO & N/A & aero & 390 & 3.75 & 23 \\
X4 ${ }^{\text {e. }}$ & M4 & None & 0 & xero & 5.60 & - & - \\
HG1-1 & M1 & TMO & 4 & aero & 328 & 3.42 & 26 \\
HG1-2 & M1 & TMO & 4 & xero & 73 & 0.04 & 2.9 \\
HG2-1 & M2 & PO & 5 & aero & 238 & 1.44 & 22 \\
HG2-2 & M2 & PO & 5 & xero & 91 & 0.04 & 3.1 \\
HG2-3 & M2 & PO & 3 & aero & 184 & 1.22 & 21 \\
HG3-1 & M3 & PO & 2 & aero & 351 & 3.02 & 29 \\
HG3-3 & M3 & PO & 0.5 & aero & 121 & 0.59 & 15 \\
HG4-1 & M4 & PO & 6 & xero & 184 & 0.07 & 2.2 \\
HG4-2 & M4 & PO & 3 & xero & 37 & 0.03 & 3.5 \\
HG5-2 & M5 & PO & 5 & xero & 291 & 0.15 & 2.5 \\
HG5-3 & M5 & PO & 3 & xero & 230 & 0.11 & 2.6 \\
\hline
\end{tabular}

a. BET surface area. ${ }^{\text {b. }}$ BJH cumulative adsorption pore volume, ${ }^{\text {c. }}$ BJH average pore diameter. ${ }^{\text {d. }}$ Value taken from ref. 5.. ${ }^{\text {e. }}$ Value taken from ref. 9.

Comparison of materials prepared with similar composition and structure, but prepared with monosilsesquioxanes vs. bissilsesquioxanes, leads to some interesting observations. For hybrid aerogels, surface areas ranged from $121-352 \mathrm{~m}^{2} / \mathrm{g}$. Interestingly, the surface area and pore diameter of HG3-1, prepared using the bissilsesquioxane M3, were larger than any of the aerogels, made with monosilsesquioxanes M2 or M1, despite having a greater fraction of alkyl 
group functionality in the final material. The larger surface area and pore diameter of HG3-1 may be indicative of the bridging nature of the alkyl functionality versus the terminal nature of the alkyl groups. The smaller surface area and pore volume observed in monosilsesquioxane materials may be a result of the alkyl groups protruding into and partially filling the mesopores. Such a phenomenon would be less likely for materials with similar alkyl functionality, but prepared with bissilsesquioxanes that can incorporate the alkyl functionality into the networks at both ends.

In general, the same trend can be noted for xerogel materials. With the exception of HG42, the surface area of all hybrid materials prepared with bissilsesquioxanes is higher than similar compositional materials made with monosilsesquioxanes. In fact, comparison of xerogel HG5-2, (prepared with bissilsequioxane M5) with xerogel HG2-2 (prepared with monosilsesquioxane M2) shows that the surface area and pore volumes of the two materials are very different, with HG5-2 having significantly larger values for both parameters. The effect on the pore diameter is not as noticeable as in the aerogel materials due to the much smaller pore size of the dense, glass-like xerogel materials. Perhaps a better comparison is that of HG5-3 to HG2-2. Even though HG5-3 has approximately twice as much Si as HG2-2, it has a similar number of alkyl groups. Its pore volume and surface area, however, only decrease slightly from those of HG5-2 and remain well above the values for HG2-2. In this case, doubling the silsesquioxane content only slightly perturbs the pore properties, suggesting a higher level of integration into the network than that observed for monosilsesquioxanes with the same alkyl chain length.

Another trend that further elucidates the nature of the network interpenetration is the decrease of the surface area and pore volume of both hybrid xerogels and aerogels as the $\mathrm{Fe} / \mathrm{Si}$ ratio decreases. For example, surface areas and pore volumes of xerogels HG4-1 and HG4-2 are smaller than the reference pure iron (III) oxide xerogel, XFe, but larger than the pure bissilsesquioxane xerogel X4. Compared to xerogel HG4-2, HG4-1 shows an even larger surface area and pore volume. The same trend is also noted for xerogels HG5-2 and HG5-3. Aerogel HG3-3 also shows this significant decrease in surface area and pore size, which is 
expected as the hybrid becomes more enriched with bissilsequioxane instead of iron (III) oxide. These differences can be attributed to the degree of interpenetration between the two networks as the $\mathrm{Fe} / \mathrm{Si}$ ratio approaches the two extremes of pure iron (III) oxide materials or pure silsesquioxane materials.

Infrared Spectroscopy. Infrared spectra of xerogels HG4-2 and HG5-3 are shown in Figure 3. The spectra of the hybrid gels show an absorption at c.a. $1080-1040 \mathrm{~cm}^{-1}$ corresponding to $v_{\text {as }}(\mathrm{Si}-\mathrm{O}-\mathrm{Si})$ from the silsesquioxane groups. ${ }^{7,19}$ Due to the presence of alkyl groups in the processed hybrid materials, these spectra also show the characteristic $\mathrm{C}-\mathrm{H}$ absorptions (c.a. 2800-3100 $\mathrm{cm}^{-1}$ ) for HG3, HG4 and HG5 gels and the C-F absorptions (c.a. $1166-1200 \mathrm{~cm}^{-1}$ ) for the HG5 gels. Features in the spectra that occur lower than $800 \mathrm{~cm}^{-1}$ are assigned to $\mathrm{Fe}-\mathrm{O}$ absorptions due to their presence in the spectra of the pure iron oxide xerogel, XFe. The bands at c.a. 680 and $470 \mathrm{~cm}^{-1}$ are common in the IR spectra of ferrihydrite, a common, amorphous polymorph of iron oxide that typically exhibits poor crystallinity. ${ }^{6 a, 20}$

The hybrid gels also show a weak, broad feature in the range of $910-940 \mathrm{~cm}^{-1}$. Metal oxide-silica composites often show a single broad band between $900-1000 \mathrm{~cm}^{-1}$ that is commonly attributed to the presence of $\mathrm{Si}-\mathrm{O}-\mathrm{M}$ bonding in the mixed oxide materials. ${ }^{19,21,22}$ This shift is often hard to assign, however, due to the presence of hydrogen bonded $\mathrm{Si}-\mathrm{OH}$ absorptions also present in the region. Often, a shift to lower energies is noted for Si-O-M absorptions in the metal silica composites as compared to the $\mathrm{Si}-\mathrm{OH}$ absorptions in the native silica materials. ${ }^{19,22}$ The shift to lower energies is used as a means to characterize the incorporation of the heavy metal atom into a $\mathrm{Si}-\mathrm{O}-\mathrm{M}$ bond. In the present case, the hybrid materials do show a shift in this band $\left(910-940 \mathrm{~cm}^{-1}\right)$ that is lower than the commonly accepted shift of $\sim 960-990 \mathrm{~cm}^{-1}$ for $\mathrm{Si}-\mathrm{OH}$ bands. This assignment however, is made difficult by the absence of $\mathrm{Si}-\mathrm{OH}$ bands in spectra of the native $\mathrm{X} 4$ and $\mathrm{X} 5$ polysilsesquioxane materials (not shown), as well as the broad nature of the peaks due to the amorphous character of the hybrids. Furthermore, this region is partially obscured by the broad absorption and shift to lower energies 

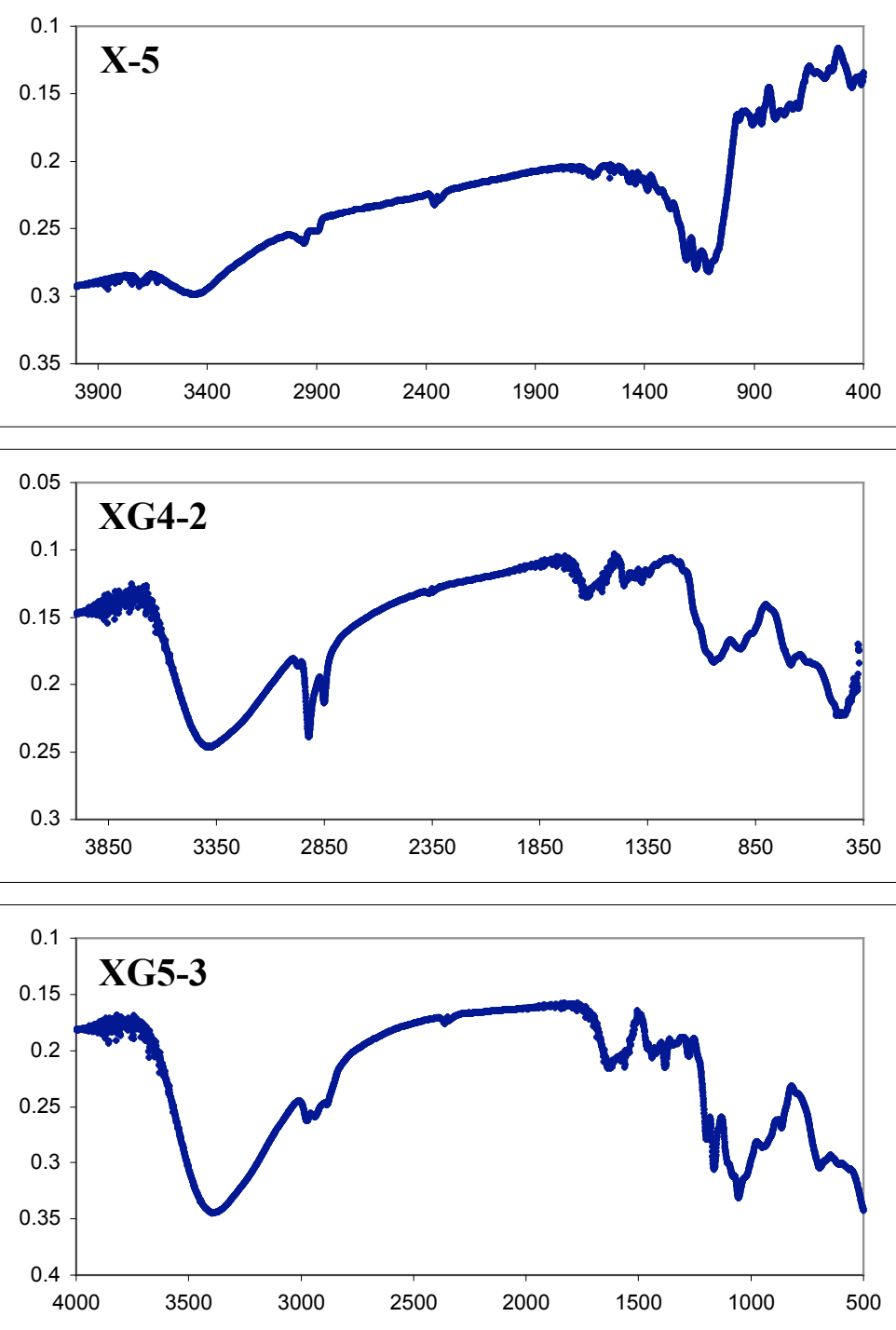

Figure 3. IR spectra of X-5, XG4-2 and XG5-3.

of the $v_{\text {as }}(\mathrm{Si}-\mathrm{O}-\mathrm{Si})$ band as the iron content increases in the gels. ${ }^{7,21}$ Although some spectroscopic evidence for the existence of $\mathrm{Si}-\mathrm{O}-\mathrm{Fe}$ bonding is present in these hybrid materials, in the absence of quantitative information the data at present indicates that the amount of $\mathrm{Si}-\mathrm{O}-\mathrm{Fe}$ bonding ${ }^{22}$ may be minor. If this is the case, the presence of two distinctly separate, interpenetrating oxide networks is indicated.

Electron Microscopy. Microscopy further supports the presence of two polymer networks that are simultaneously formed and highly interpenetrated with each other to generate these 
hybrid nanocomposites. The surface morphology and microstructure of selected hybrid gels was evaluated with SEM. The pure, single network control xerogels, XFe and X4, were used for comparison to the hybrid, IPN xerogels. Figure 4 contains SEM images of three xerogels: XFe, X4 and HG4-3. The images reveal the highly porous nature of these materials, which was also established by $\mathrm{N}_{2}$ adsorption/desorption analysis. Although the SEM image of HG4-3 exhibits differences in the surface texture from the iron oxide XFe and bridged polysilsesquioxane $\mathbf{X} \mathbf{4}$ gels, the surface morphology appears uniform without obvious phase separation between the two networks. No difference in morphology was noted between the surfaces of IPN materials prepared with monosilsesquioxanes (HG1 and HG2; images not shown) and bissilsesquioxanes.

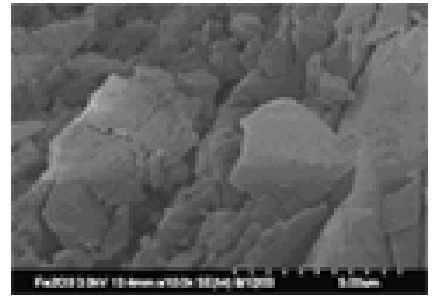

(a) XFe

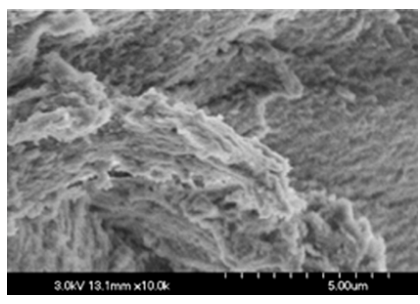

(b) $\mathrm{X} 4$

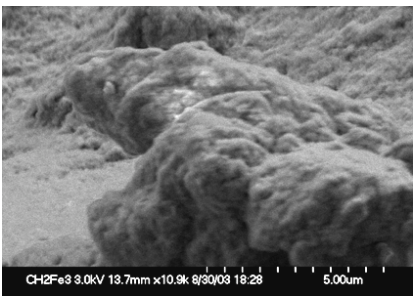

(c)HG4-3

Figure 4. SEM images of (a) iron (III) oxide xerogel (XFe), (b) polysilsesquioxane xerogel (X4), and (c) hybrid xerogel (HG4-3)

The nanostructure of the hybrids gels using HRTEM and EFTEM was probed to evaluate the hybrid gels' architechture on smaller length scales. Figure 5 contains HRTEM and EFTEM images of areogel HG2 $(\mathrm{Fe} / \mathrm{Si}=5), \mathbf{H G 3}(\mathrm{Fe} / \mathrm{Si}=2)$, and xerogel HG5-3 $(\mathrm{Fe} / \mathrm{Si}=3)$. According to these images, these materials are a collection of nanoparticles interconnected to form an amorphous, mesoporous structure. The size of the particles appears to be fairly uniform throughout the gels, with particles sizes ranging from 2-5 nm in diameter. No evidence exists for distinct phase separation between Fe- and Si-oxide domains at length scales $>5 \mathrm{~nm}$, further supporting the conclusions drawn from the SEM images. Furthermore, the observed uniformity is confirmed by the element maps of HG2 (fluorine map) and HG3 ( Fe, Si, and C maps), as 
(a)

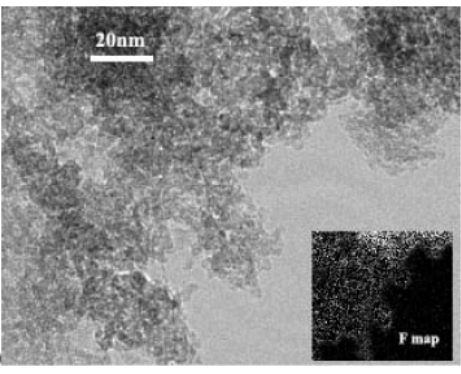

(b)

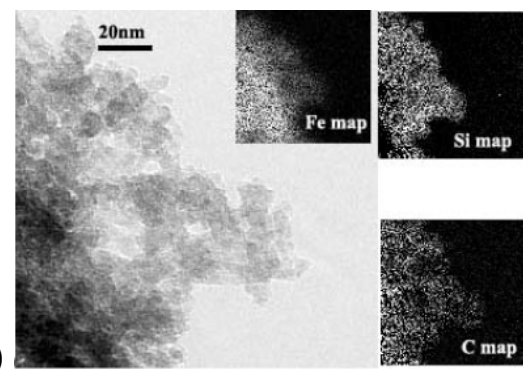

$10 \mathrm{~nm}$

(c)

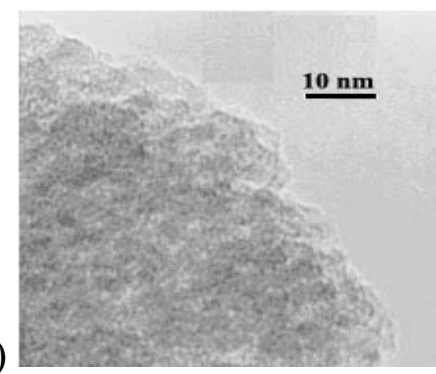

Figure 5. TEM and EFTEM micrographs of hybrid gels (a) aerogel HG1, (b) aerogel HG3, ( c) xerogel HG5-3.

shown in the insets of Figure $4 \mathrm{a}$ and $4 \mathrm{~b}$ respectively. All element maps show a high degree of mixing between the iron oxide and polysilsesquioxane phases on the nanoscale as well as the microscale. Microscopy data further support the formation of two polymer networks that are simultaneously formed and highly interpenetrated with each other.

Ultra Small Angle X-ray Scattering (USAXS). Ultra small-angle X-ray scattering (USAXS) is a technique used for microstructural characterization. Many complex materials are built up by hierarchical assembly. USAXS extends the observable length scale of features of these hybrid materials from 2 to $10,000 \AA$ (Figure $6 a, 6 b) .{ }^{23}$ USAXS is primarily sensitive to the iron atoms since the scattering power is proportional to atomic number. The data are dominated by intense scattering at small scattering vectors $(q)$, which is pure power-law in form. This intense scattering is due to the powder granules and could be masking morphological features in the $100-10,000 \AA$ range. In addition to the scattering from the powder itself, additional scattering is seen at $q>0.01 \AA^{-1}$, corresponding to scattering from structures that are smaller than $100 \AA$. Such scattering is characteristic of dried gels as previously seen for bridgedsilsesquioxane derived aerogels and xerogels. ${ }^{24}$

To better visualize the scattering from the gel structures, the intense powder scattering is subtracted from the data and the results are shown in Figure 6b. The USAXS data are scaled to match to $0.5 \AA^{-1}$. For the iron oxide xerogel, XFe, a single structure level with a radius of gyration of $26.5 \AA(2.65 \mathrm{~nm})$ is observed. We suggest that it represents the radius of the 

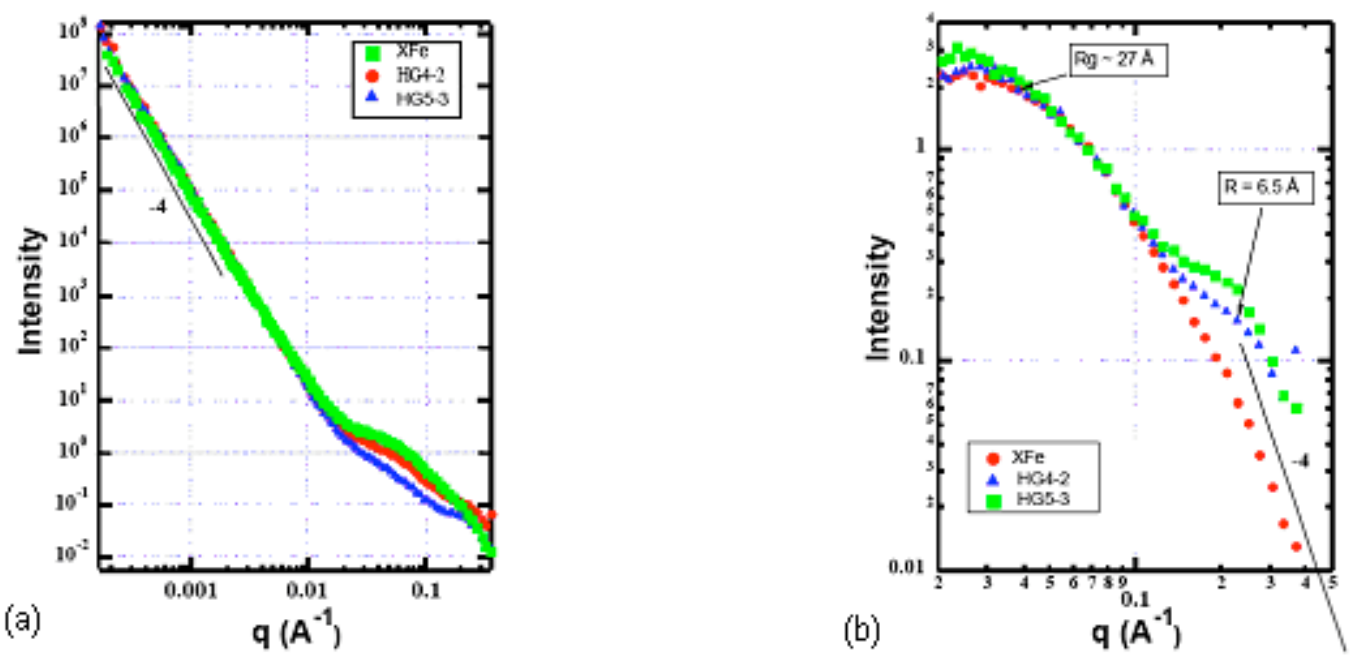

Figure 6. USAXS data for xerogels XFe, HG4-2, and HG5-3. Data are not directly comparable in intensity since the amount of sample in the beam is unknown. (a) USAXS data with intense power-law scattering at small-q subtracted. (b) USAXS data scaled to match at $0.5 \AA^{-1}$. The $R g$ value reported is the Guinier radius, which is the radius of gyration of the scattering domains.

nanoparticles that build the gel's skeletal structure, which is consistent with the TEM data that also showed particle sizes to be in this same size regime. The limiting slopes seem to approach -4 , characteristic of a smooth interface. This feature is also shown in the data for hybrid materials HG4-2 and HG5-3.

Table 4 shows the actual length scales measured by fitting the data to standard models. The results suggest that, when hybrid gels are compared to pure iron(III) oxide gels, the iron oxide network mesh is unchanged by the presence of polysilsesquioxane. Because of the similarity on the scattering from the $26 \AA$ feature, it is reasonable to attribute this feature to the same iron oxide network found in the absence of a silica related component. However, it cannot be ruled out that this feature arises from a silica-iron(III) oxide-like mixed phase.

In summary, the USAXS data, provides no evidence of large-scale $(5 \mathrm{~nm})$ phase separation consistent with the HRTEM and EFTEM results discussed above. At best, tiny $6 \AA$ silica domains may exist, as evidenced by the presence of a second feature about $6 \AA$ in radius- 
Table 4. Guinier radius values $\left(R_{\mathrm{g}}\right)$ of Iron xerogel and hybrid xerogel materials

\begin{tabular}{ccc}
\hline Sample & Level1 $R_{\mathrm{g}}(\AA)$ & Level2 $R_{\mathrm{g}}(\AA)$ \\
\hline XFe & --- & 26.5 \\
HG4-2 & 6.3 & 28.2 \\
HG5-3 & 6.9 & 31.5 \\
\hline
\end{tabular}

of-gyration also present in the hybrid materials. Alternatively, the $6 \AA$ feature may be the strut size (average distance of bridging alkyl or perfluoroalkyl group) of an interpenetrating silsesquioxane network. Finally, the $6 \AA$ feature could be a broadened Bragg peak corresponding to organization of the organic groups of polysilsesquioxane component. In this case the periodicity would be approximately $(2 / 0.2) \AA \approx 30 \AA$. However there is no corroborating evidence for this at present. These results call attention to the difficulty in distinguishing between the IPN's integrated at molecular length scales and copolymers.

\section{Conclusions}

We have established a sol-gel approach for the synthesis of organic/inorganic hybrid nanocomposites containing iron oxide and polysilsesquioxanes. The synthetic procedure allowed for the simultaneous formation iron (III) oxide and bridged polysilsesquioxane networks from $\mathrm{FeCl}_{3} \cdot 6 \mathrm{H}_{2} \mathrm{O}$ and functionalized silsesquioxane monomers in a one-pot reaction. The resulting interpenetrating networks resulted in monolithic hybrid xerogels or aerogels when using organic epoxides as gelation agents. The two components were highly or even molecularly interpenetrated to provide the integrated chemical systems. According to the characterization results for the nanostructures, as well as the physical properties of materials, the hybrid nanocomposites exhibited a uniform dispersion of both components with no evidence for phase separation on length scales $>5 \mathrm{~nm}$. This method provides a general synthetic method for the synthesis of a variety of organic/inorganic hybrid nanocomposites using a common metal salt 
and functionalized silane monomers. We believe that this methodology is general for the preparation of a large variety of transition and main group metal hybrid composites. ${ }^{25}$

\section{Acknowledgement}

This work was performed under the auspices of the US Department of Energy by the University of California, Lawrence Livermore National Laboratory under contract No. W-7405Eng-48. Special thanks go to Jennifer S. Harper and Dr. Wen-An Chiou for TEM and EFTEM analyses. We thank Jan Ilavsky for his guidance in using the USAXS instrument at UNICAT and for development of the analysis software. The UNICAT facility is supported by the U.S. DOE under Award No. DEFG02-91ER45439 (University of Illinois at Urbana-Champaign), the Oak Ridge National Laboratory (U.S. DOE Contract DE-AC05-00OR22725), the National Institute of Standards and Technology (U.S. Department of Commerce) and UOP LLC. Lihua Zhao wishes to thank LLNL for a graduate student research fellowship.

\section{References:}

1. (a) Sanchez, C.; Soler-Illia, G. J. de A. A.; Ribot, F.; Lalot, T.; Mayer, C. R.; Cabuil, V. Chem. Mater. 2001, 13, 3061 and references therein. (b) Loy, D. A.; Shea, K. J. Chem. Rev. 1995, 95, 131 and references therein.

2. Klempner, D.; Sperling, L. H.; Utracki, L. A. Interpenetrating polymer networks; American Chemcial Society, Washington, DC, 1994.

3. (a) Sperling, L. H. An Overview of Interpenetrating Networks in Polymeric Materials Encyclopedia; Joseph C. Salamone, Ed ed.; CRC Press:Boca Raton, FL, 1996; Vol. 5 and references therein. (b) Baidak, A. A.; Liegeois, J. M.; Sperling, L. H. J. Polym. Sci., Part B:Polym. Phys. 1997, 35, 1973-1984.

4. (a) Vlad, S.; Vlad, A.; Oprea, S. Europ. Polym. J. 2002, 38, 829. (b) Ting, R. Y.; Capps, R. N.; Klempner, D. Abstr. Pap.- Am. Chem. Soc. 1989, 197, 143. (c) Gettlema, L.; Farris, C. L.; Rawls, H. R.; LeBouef, R. J. U. S. Patent 1985; 4,543,379. 
5. Gash, A. E.; Tillotson, T. M.; Satcher, J. H. Jr.; Poco, J. F.; Hrubesh, L. W.; Simpson, R. L. Chem. Mater. 2001, 13, 999.

6. (a) Gash, A. E; Satcher J. H. Jr.; Simpson R. L. Chem. Mater. 2003, 15, 3268. (b) Tillotson, T. M.; Gash, A. E.; Simpson, R. L.; Hrubesh, L. W.; Satcher, J. H. Jr.; Poco, J. F. J. Non-Cryst. Solids 2001, 285, 338.

7. Clapsaddle, B. J.; Gash. A. E.; Satcher, J. H. Jr.; Simpson, R. L. J. Non-Cryst. Solids, 2003, 331(1-3), 190.

8. Purification of Laboratory Chemicals, $4^{\text {th }}$ Edition, Amarego Perrin.

9. Oviatt, H. W.; Shea, K. J.; Small, J. H. Chem. Mater. 1993, 5, 943.

10. Církva, V.; Gaboyard, M.; Paleta, O. J. Fluorine Chem. 2000, 102, 349.

11. Manseri, A.; Ameduri, B.; Boutevin, B.; Kotora, M.; Hajek, M.; Caporiccio, G. J. Fluorine Chem. 1995, 73, 151.

12. Ameduri, B.; Boutevin, B.; Moreau, J. J. E.; Moutaabbid, H.; Man, M. W. C. J. Fluorine Chem. 2000, 104, 185.

13. Knochel, P.; Chang, M.; Yeh, P.; Berk, S. C.; Talbert, J. J. Org. Chem. 1988, 53, 2390.

14. Shea, K. J.; Loy, D. A.; Webster, O. J. Am. Chem. Soc. 1992, 114, 6700.

15. (a) Brunauer, S.; Emmett, P. H.; Teller, E. J. J. Am. Chem. Soc. 1938, 60, 309. (b) Gregg, S. J.; SingK. S. W. Adsorption, Surface Area, and Porosity, $2^{\text {nd }}$ Ed.; Academic Press: London, 1982.

16. Barrett, E. P.; Joyner, L. G.; Halenda, P. P. J. Am. Chem. Soc. 1951, 73, 373.

17. Loy, D.A.; Straumanis, A.; Schneider, D. A.; Mather, B.; Sanchez, A.; Baugher, C. R.; Shea, K. J. Polymer ,Preprints (American Chemical Society, Division of Polymer Chemistry), 2004, 45(1), 591.

18. (a) Loy, D.A.; Mather, B.; Straumanis, A. R.; Baugher, C.; Schneider, D. A.; Sanchez, A.; Shea, K. J. Chem. Mater. 2004, 16(11), 2041. (b) Jolivet, J.P.; Chanéac, C.; Tronc, E. Chem. Commun. 2004, (5), 481. 
19. Maxim, N.; Overweg, A.; Kooyman, P. J.; Wolput, J. H. M. van; Hanssen, R. W. J. M.; van Santen, R. A.; Abbenhuis, H. C. L. J. Phys. Chem. 2002, 106, 2203.

20. (a) Cornell, R. M.; Schwertmann, U. The Iron Oxides; VCH Publishers: Weinheim, 1996. (b) Schwertmann, U.; Cornell, R. M. Iron Oxides in the Laboratory; VCH Publishers: New York, 1991.

21. Fabrizioli, P.; Burgi, T.; Bergener, M.; van Doorslaer, S.; Baiker, A. J. Mater. Chem. 2002, 12, 619 .

22. Gao, X.; Bre, S. R.; Fierro, J. L. G.; Banares, M. A.; Wachs, I. E. J. Phys. Chem. B 1998, $102,5653$.

23. Matsuoka, H.; Kakigami, K.; Ise, N.; Kobayashi,, Y.: Machitani, Y.; Kikuchi, T.; Kato, T. Proc. Nat. Acad. Sci. USA 1991, 88, 6618.

24. Schaefer, D. W.; Beaucage, G.; Loy, D. A.; Shea, K. J.; Lin, J. S. Chem. Mater. 2004, 16(8), 1402.

25. Clapsaddle, B.J.; Sprehn, D. W.; Gash, A. E.; Satcher, J. H. Jr.; Simpson, R. L. J. NonCryst. Solids 2004, in press. 